\title{
Delay Tracking Algorithm for the Pilot Component of GLONASS Perspective Signal with Code Division Multiple Access
}

\author{
Alexandr Ivanovich Perov ${ }^{1}$ \\ ${ }^{1}$ National Research University, Moscow Power Engineering Institute, Russia \\ Correspondence: Alexandr Ivanovich Perov, National Research University, Moscow Power Engineering Institute, \\ Krasnokazarmennaya, 14, 111250, Moscow, Russia. E-mail: alexp@aha.ru
}

Received: February 6, 2015

Accepted: February 13, $2015 \quad$ Online Published: March 25, 2015

doi:10.5539/mas.v9n5p144

URL: http://dx.doi.org/10.5539/mas.v9n5p144

\begin{abstract}
The paper studies the issue of the synthesis of the optimal code delay tracking algorithm for the pilot component of GLONASS perspective signal with $\mathrm{BOC}(1,1)$ modulation and processing at subcarrier frequencies.

The study objective is synthesis and analysis of optimal code delay tracking algorithm of $\operatorname{BOC}(1,1)$ modulated signal (pilot component of L1OC GLONASS perspective signal) using the method of additional variable and processing at subcarrier frequencies.

Equations describing optimal tracking system with account to multimodality of code delay aposteriori probability density are calculated using the theory of optimal filtering and the method of additional variable.

The structural scheme of delay discriminator with processing at subcarrier frequencies is developed. Discrimination and fluctuation characteristics of synthesized delay discriminator are calculated. The results of the proposed tracking algorithm modelling, which demonstrate its performance and accuracy characteristics, are presented.
\end{abstract}

Keywords: satellite radio navigation system, consumer equipment, tracking system, modulation, subcarrier frequency, statistical characteristics, accuracy, optimal filtering

\section{Introduction}

\subsection{Introduce the Problem}

Since 2012 the Federal Target Program of "GLONASS Maintenance, Development and Use for 2012 - 2020 years" is effective in the Russian Federation, based upon which beginning from 2016 new GLONASS signals with code division will be emitted from satellite vehicles GLONASS-K2. In L1, L2 frequency ranges the signals of open access are two-component signals containing pilot and information components, the combining of which into a single navigation signal is carried out according to the method of by-bit time multiplexing (Perov \& Harisov, 2010). The information component of this signal has standard binary modulation (BPSK) with a frequency of code ranging symbols of $1,023 \mathrm{MHz}$, and the pilot component has modulation at subcarrier frequencies $\operatorname{BOC}(1,1)$ with a base frequency of $f_{b}=1,023 \mathrm{MHz}$.

It is planned to use signals with similar type of modulation in modernizing navigation satellite system GPS (Interface Specification GPS Space Segment/ User Segment. IS-GPS-200F, 21 September 2011; Interface specification GPS Space Segment/User Segment L1C Interfaces. IS-GPS-800B, 21 September 2011) and in advanced navigation system (European GNSS (GALILEO), 2010; BeiDou, 2011).

\subsection{Importance of the Problem}

BOC-modulation signals tracking has a number of peculiarities associated with the envelope correlation function's several extremums. It leads to the multimodality nature of signal delay aposteriori probability density and there is a problem of possible ambiguity of delay estimation. That is why it is relevant to develop BOC-modulation signal detection and processing algorithms with regard to aposteriori distribution multimodality.

\section{Literature Review}

A number of studies are concerned with this topic. Martin N. et al. study the problem of BOC-modulation signal acquisition, the algorithm, in which BOC-modulation signal is considered as a sum of two signals with BPSK 
modulation at subcarrier frequencies symmetrically located in relation to carrier frequency is described. Heiries V. et al. give the results of this method used to detect signals with BOC $(10,5)$ and BOC $(14,2)$ modulations, which shows their high efficiency. Chen S. et al. (Chen S. et al., 2006) bring the ideology of BOC modulation signal presentation in the form of two signals with BPSK modulation at subcarrier frequencies, it is used to track signal delay and phase. However, proposed processing algorithms are heuristic; they do not result from a strict synthesis.

Fante R. L., David de Castro et al. study signal delay estimation algorithms, wherein besides "early" and "late" reference signals "very early" and "very late" signals are additionally used. Such approach allows extending the discriminator curve of delay discriminator, but does not solve the problems of the multimodality of aposteriori probability density.

Some of modifications of this approach with respect to various types of BOC-modulations have been described (Musso et al., 2006; Jovancevic et al., 2007; Gyu-In et al., 2007; Jovanovic et al, 2012).

Hodgart et al. (Hodgart et al, 2007; Hodgart et al., 2008) studied BOC modulation signals tracking algorithms with separate signal delay tracking systems related to BPSK ranging code and signal delay related to a digital subcarrier. In such a manner, two delay estimations are united into final estimation according to the corresponding algorithm. It shall be noted that such approach known as "additional variable" was described by Tikhonov V. I. and Harisov V. N. in 1984 and was widely used for signal delay tracking problem solving with extraction delay information from the envelope and signal phase (Perov, A. I., \& Harisov, V. N., 2010; Kaplan, E. D., 2006). Its main intended use is disambiguation of estimations driven by the estimated parameter aposteriori probability density multimodality.

As distinct from Hodgart et al. (Hodgart et al., 2007; Hodgart et al., 2008) Tikhonov, V. I. and Harisov V. N. (Tikhionov, V. I., Kharisov, V. N., 2004) used two components tracking (main and additional variables) which is carried out in one tracking loop. It shall be noted that this fact results from the rigorous theory of optimal system filtration synthesis.

Vinogradov et al. (Vinogradov et al., 2006) used the given approach to solve BOC modulation signal delay filtration problems.

In the articles mentioned, the processing of received signals is carried out at carrier frequency using the classic signal phase tracking system. It has been mentioned when receiving BOC modulation signals the processing at subcarrier frequency is possible, that may offer certain advantages.

The objective of the article is the synthesis and analysis of optimal code delay tracking algorithm with BOC(1,1) modulation (L1OC GLONASS signal testing component) using the method of additional variable and with processing at subcarrier frequencies.

\section{Method}

\subsection{Setting the Synthesis Problem}

We use optimal filtering theory to synthesize optimal (quasioptimal) tracking system, which implies statistical description of processes and observations. Let us assume there is an additive mixture of navigation signal $s(t)$ and receiver internal noise $n(t)$ at the receiver input, the noise is assumed to be white Gaussian with one-way power spectral density $N_{0}$.

L1OC GLONASS signals with code division, which is a two-component signal containing pilot and information components, is considered as a navigation signal (Perov, A. I., \& Harisov, V. N., 2010).

The pilot component $s_{p}(t)$ has BOC $(1,1)$ modulation with a base frequency of $f_{b}=1,023 \mathrm{MHz}$ and is designed for delay, phase, and Doppler frequency shift measuring. The information component $s_{d}(t)$ has BPSK(1) modulation and contains digital information (navigation message). The combination of two components into a single radio signal is carried out on the principle of by-bit time multiplexing.

It is assumed that sampling of input process by time is carried out in the receiver, thus realization in discrete time $t_{k, i}$ enters signal-processing system (Figure 1)

$$
y_{k, i}=s\left(t_{k, i}, \varphi_{k, i}\right)+n_{k, i}
$$

where $t_{k, i}=k T+i T_{d} ; T=N T_{d}$ - discrete processing step in tracking system loop; $T_{d}$ - sampling period 
in ADC; $\varphi_{k, i}$ - signal phase subject to estimation; $n_{k, i}$ - discrete white Gaussian noise with a dispersion of $\sigma_{n}^{2}=\frac{N_{0}}{2 T_{d}}$, where $N_{0}-$ receiver internal noise one-way power spectral density.

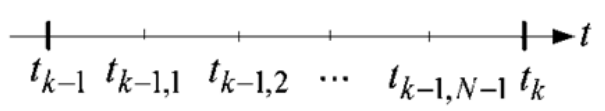

Figure 1. Indexing time scheme

In the paper double time indexing is used, which is necessary for the purpose of correct accounting of input counts $y_{k, i}$ processing in correlators and rarer processing (with a time step $T$ ) in tracking system smoothing filter when synthesizing the tracking system.

It is supposed to form estimations of filtered process $\varphi_{k}$ in clock time moments $t_{k}, k=1,2, \ldots$ such that $t_{k}-t_{k-1}=N T_{d}=T, t_{k-1, N}=t_{k}, t_{k-1,0}=t_{k-1}$.

The paper studies the synthesis of the tracking system, which implies retrieval of information only from pilot component of L1OC signal. To carry out this synthesis we write signal L1OC $s\left(t_{k, i}, \varphi_{k, i}\right)$ in (1) in the following form

$$
s\left(t_{k, i}, \varphi_{k, i}\right)=s_{p}\left(t_{k, i}, \varphi_{k, i}\right) \otimes s_{d}\left(t_{k, i}, \varphi_{k, i}\right),
$$

where sign $\otimes$ means an operation of by-bit time multiplexing;

$$
s_{p}\left(t_{k, i}, \varphi_{k, i}\right)=A h_{c, p}\left(t_{k, i}-\tau_{k}\right) h_{d s}\left(t_{k, i}-\tau_{k}\right) \cos \left(\omega_{0} t_{k, i}+\varphi_{k, i}\right)
$$

— signal pilot component;

$$
s_{d}\left(t_{k, i}, \tau_{k}\right)=A h_{c, d}\left(t_{k, i}-\tau_{k}\right) h_{D I}\left(t_{k, i}-\tau_{k}\right) \cos \left(\omega_{0} t_{k, i}+\varphi_{k, i}\right)
$$

— signal information component;

$A$ - signal amplitude; $\tau_{k}-$ signal delay; $h_{c, p}\left(t_{k, i}\right), h_{c, d}\left(t_{k, i}\right)$ - functions of modulation with ranging codes of pilot and information components; $h_{d s}\left(t_{k, i}\right)=\operatorname{sign}\left(\sin \left(2 \pi f_{s}\right)\right)-$ function of modulation with a digital sinusoid, where $f_{s}$ - sinusoid frequency; $h_{D I}\left(t_{k, i}\right)$ - function of modulation with digital information (navigation message).

To carry out the synthesis of phase filtration optimal algorithm by the pilot component we present the signal multiplexed by time (2) as a sum of two signals

$$
\begin{gathered}
s\left(t_{k, i}, \varphi_{k, i}\right)=\tilde{s}_{p}\left(t_{k, i}, \lambda_{k}\right)+\tilde{s}_{d}\left(t_{k, i}\right), \\
\tilde{s}_{p}\left(t_{k, i}, \varphi_{k, i}\right)=A \tilde{h}_{c, p}\left(t_{k, i}-\tau_{k}\right) h_{d s}\left(t_{k, i}-\tau_{k}\right) \cos \left(\omega_{0} t_{k, i}+\varphi_{k, i}\right), \\
\tilde{s}_{d}\left(t_{k, i}\right)=A \tilde{h}_{c, d}\left(t_{k, i}-\tau_{k}\right) h_{D I}\left(t_{k, i}-\tau_{k}\right) \cos \left(\omega_{0} t_{k, i}+\varphi_{k, i}\right) .
\end{gathered}
$$

where $\tilde{h}_{c, d}\left(t_{k, i}\right)$ - sequence resulting from the sequence $h_{c, d}\left(t_{k, i}\right)$ by means of representing each symbol in 
the form of two semisymbols, the value of the former is equal to the value of the corresponding symbol $h_{c, d}\left(t_{k, i}\right)$, and the value of the latter is assumed to be zero; $\tilde{h}_{c, p}\left(t_{k, i}\right)$ - sequence resulting from the sequence $h_{c, p}\left(t_{k, i}\right)$ means of representing each symbol of ranging code in the form of two semisymbols, the value of one of which is assumed to be zero, and the value of the other is equal to the value of the corresponding symbol $h_{c, p}\left(t_{k, i}\right)$.

When writing the expression (3) we formally suppose that information component does not depend on the estimated parameter $\tau_{k}$.

Considering signal delay the estimated parameter with regard to (3), observations (1) can be written as:

$$
y_{k, i}=\tilde{s}_{p}\left(t_{k, i}, \tau_{k}\right)+\tilde{s}_{d}\left(t_{k, i}\right)+n_{k, i} .
$$

When synthesizing delay filtering quasioptimal algorithm we assume that phase and Doppler shift are independently estimated in the receiver, i.e. $\tilde{\varphi}_{k, i}$ and $\hat{\omega}_{D, k}$ are known.

In this case, the signal delay dynamic model can be written as

$$
\tau_{k}=\tau_{k-1}-\tilde{\omega}_{D, k} T /\left(2 \pi f_{0}\right)+\xi_{\tau, k-1} .
$$

where $f_{0}$ - signal carrier frequency; $\xi_{\tau, k-1}$ - discrete white Gaussian noise with a dispersion of $D_{\xi}$.

The problem of the synthesis of the quasioptimal algorithm of delay filtering $\tau_{k}$ in clock time moments $t_{k}$ when processing only the signal pilot component $\tilde{s}_{p}\left(t_{k, i}, \tau_{k}\right)$ and observations (1) with discretization interval $T_{d}$ is set.

\subsection{The Synthesis of Quasioptimal Delay Filtration Algorithm}

Where the rate of taking observations is significantly higher than the rate of $\hat{\tau}_{k}$ delay calculations, for the synthesis of optimal filtration algorithm it is necessary to use the theory of optimal filtration with grouping of observations (Perov, A. I., 2012).

Taking into account the periodicity of subcarrier fluctuation $\operatorname{sign}\left(\sin \left(2 \pi f_{s}\right)\right)$, the aposteriori density of delay probability density bears multimodal nature.

For the synthesis of quasioptimal filtration algorithm upon such conditions, we can use the additional variable method (Perov, A. I., \& Harisov, V. H., 2010; Tikhonov, V. I., Harisov, V. N., 2004).

Let us introduce additional variable $\tau_{d}$, which we will connect with the digital sinusoid of the pilot component, i.e.

$$
\tilde{s}_{p}\left(t_{k, i}, \tau_{k}, \tau_{d, k}\right)=A \tilde{h}_{c, p}\left(t_{k, i}-\tau_{k}\right) h_{d s}\left(t_{k, i}-\tau_{d, k}\right) \cos \left(\omega_{0} t_{k, i}+\varphi_{k, i}\right) .
$$

Herewith let $\tau_{d, k}=\tau_{k}$ for any $k$. Consequently, the $\tau_{d}$ in time is described with equation similar to (5). Now we shall introduce the expanded vector $\mathrm{x}=\left|\begin{array}{ll}\tau & \tau_{d}\end{array}\right|^{\mathrm{T}}$, for which we will write down the vector equation:

$$
\mathrm{x}_{k}=\mathrm{Fx}_{k-1}+\mathrm{P} u_{k}+\mathrm{G} \xi_{\tau, k-1},
$$

where: $\quad \mathrm{F}=\left|\begin{array}{ll}1 & 0 \\ 0 & 1\end{array}\right|, \quad \mathrm{G}=\left|\begin{array}{l}1 \\ 1\end{array}\right|, \quad \mathrm{P}=\left|\begin{array}{l}1 \\ 1\end{array}\right|, \quad u_{k}=-\tilde{\omega}_{D, k} T /\left(2 \pi f_{0}\right)$.

In the examined task on the time interval, $\left[t_{k-1,1}, t_{k}\right]$ (see Figure 1) the observations

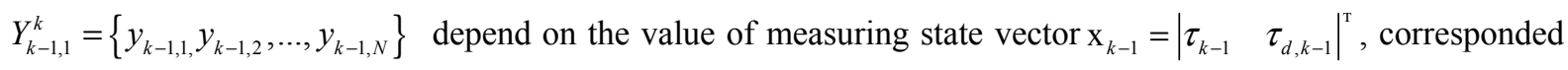


to the moment of time $t_{k-1}$. That is why, if we are interested in the current estimation of state $\mathrm{x}_{k-1}$ using observations $Y_{0}^{k}=\left\{Y_{0,1}^{1}, Y_{1,1}^{2}, \ldots, Y_{k-1,1}^{k}\right\}$, it's necessary to consider the aposteriori probability density $p\left(\mathrm{x}_{k-1} \mid Y_{0}^{k}\right)$, for which we can write down the equations:

$$
\begin{gathered}
p\left(\mathrm{x}_{k-1} \mid Y_{0}^{k}\right)=c p\left(\mathrm{x}_{k-1} \mid Y_{0}^{k-1}\right) p\left(Y_{k-1,1}^{k} \mid \mathrm{x}_{k-1}\right), \\
p\left(\mathrm{x}_{k-1} \mid Y_{0}^{k-1}\right)=\int_{-\infty}^{\infty} p\left(\mathrm{x}_{k-2} \mid Y_{0}^{k-1}\right) p\left(\mathrm{x}_{k-1} \mid \mathrm{x}_{k-2}\right) d \mathrm{x}_{k-2} .
\end{gathered}
$$

Consequently, now $t_{k}$ with all the available for this moment observations of $Y_{0}^{k}$ in process, we will form the estimation of state vector $\hat{\mathbf{x}}_{k-1}$ being correspondent to the state vector $\mathrm{x}_{k-1}$ for $t_{k-1}$ moment of time.

In accordance with the additional variable method, we firstly consider the aposteriori probability density of the expanded state vector $\mathrm{x}$ for one of the periods of periodical argument with use of standard Gaussian approximation in the theory of optimal filtration. The equations describing the change of mathematical expectation $\hat{\mathrm{x}}_{k-1}=E\left[\mathrm{x}_{k-1}\right]$ and the matrix of dispersions $\mathrm{D}_{\mathrm{x}, k-1}$ of Gaussian aposteriori probability density are called the equations of optimal (or quasioptimal - when using these or those approximations) filtration.

For the examined setting of the problem, the algorithm of quasioptimal filtration of vector $\mathrm{x}_{k-1}$ can be presented with the following equations (Tikhionov, V. I., \& Kharisov, V. N., 2004):

$$
\begin{gathered}
\hat{\mathrm{x}}_{k-1}=\tilde{\mathrm{x}}_{k-1}+\mathrm{D}_{\mathrm{x}, k-1}\left(\frac{\partial \tilde{F}_{k}\left(\tilde{\mathrm{x}}_{k-1}\right)}{\partial \mathrm{x}}\right)^{T}, \tilde{\mathrm{x}}_{k-1}=\mathrm{F} \hat{\mathrm{x}}_{k-2}+\mathrm{P} u_{k-1}, \\
\tilde{\mathrm{D}}_{\mathrm{x}, k-1}=\mathrm{FD}_{\mathrm{x}, k-2} \mathrm{~F}^{T}+\mathrm{G} D_{\xi} \mathrm{G}^{T}, \mathrm{D}_{\mathrm{x}, k-1}^{-1}=\tilde{\mathrm{D}}_{\mathrm{x}, k-1}^{-1}-\frac{\partial}{\partial \mathrm{x}}\left(\frac{\partial \tilde{F}_{k}\left(\tilde{\mathrm{x}}_{k-1}\right)}{\partial \mathrm{x}}\right)^{T},
\end{gathered}
$$

where $\hat{\mathrm{x}}_{k-1}$ - valuation of filtered process $\tilde{\mathrm{x}}_{k-1}-$ extrapolate valuation of process; $\mathrm{D}_{\mathrm{x}, k-1}-$ matrix of errors filtration dispersion; $\tilde{\mathrm{D}}_{\mathrm{x}, k-1}$ - matrix of extrapolate errors dispersion;

$$
\tilde{F}_{k}\left(\mathrm{x}_{k-1}\right)=\frac{1}{\sigma_{n}^{2}} \sum_{i=1}^{N} y_{k-1, i}\left(\tilde{s}_{p}\left(t_{k-1, i}, \mathrm{x}_{k-1}\right)+\tilde{s}_{d}\left(t_{k-1, i}\right)\right) .
$$

As $\tilde{s}_{d}\left(t_{k-1, i}\right)$ does not depend upon estimated state vector $\mathrm{x}_{k-1}$, for further examination suggesting differentiation functions $\tilde{F}_{k}\left(\mathrm{x}_{k-1}\right)$ on $\mathrm{x}_{k-1}$, we will consider the simplified form:

$$
\tilde{F}_{k}\left(\mathrm{x}_{k-1}\right) \approx \frac{1}{\sigma_{n}^{2}} \sum_{i=1}^{N} y_{k-1, i} \tilde{s}_{p}\left(t_{k-1, i}, \mathrm{x}_{k-1}\right) .
$$

Now we shall modify the right part of the formula (11)

$$
\begin{gathered}
\frac{1}{\sigma_{n}^{2}} \sum_{l=1}^{N_{p}} y_{k-1, l} \tilde{s}_{p}\left(t_{k-1, l}, \mathrm{x}_{k-1}\right)= \\
=\frac{A}{\sigma_{n}^{2}} \sum_{l=1}^{N} y_{k-1, l} \tilde{h}_{c, p}\left(t_{k-1, l}-\tau_{k-1}\right) h_{d s}\left(t_{k-1, l}-\tau_{d, k-1}\right) \cos \left(\omega_{0} t_{k-1, l}+\tilde{\varphi}_{k-1}, l\right)= \\
=\frac{A}{\sigma_{n}^{2}} \sum_{l=1}^{N} y_{k-1, l} \tilde{h}_{c, p}\left(t_{k-1, l}-\tau_{k-1}\right) h_{d s}\left(t_{k-1, l}-\tau_{d, k-1}\right) \cos \left(\omega_{0} t_{k-1, l}+\tilde{\varphi}_{k-1, l}\right),
\end{gathered}
$$

where $\tilde{\varphi}_{k-1, l}=\hat{\varphi}_{k-1}+l T_{d} \hat{\omega}_{D, k-1}$ - extrapolated estimation of signal phase. 
The present expression can be interpreted as the correlation processing of incoming counts $y_{k-1, l}$ with reference signal $\tilde{s}_{p}\left(t_{k-1, l}, \mathrm{x}_{k-1}\right)$, which shall be formed in the point of extrapolated estimation $\mathrm{x}_{k-1} \rightarrow \tilde{\mathrm{x}}_{k}$, what supposes the processing at carrier frequency $\omega_{0}$. Thus, the present formula does not contain subcarrier frequencies in explicit form, what will allow transiting to the processing at these frequencies. To provide the possibility of such processing we will consider the signal function $\tilde{s}_{p}\left(t_{k, i}, \tau_{k-1}\right)$ (4) and interchange (in the first approximation) $\operatorname{sign}\left(\sin \left(\Omega t_{k-1, l}\right)\right)$ to $\sin \left(\Omega t_{k-1, l}\right)$ :

$$
\tilde{s}_{p}\left(t_{k-1, l}, \mathbf{x}_{k-1}\right)=A \tilde{h}_{c, p}\left(t_{k-1, l}-\tau_{k-1}\right) \sin \left(\Omega\left(t_{k-1, l}-\tau_{d, k-1}\right)\right) \cos \left(\omega_{0} t_{k-1, l}+\tilde{\varphi}_{k-1, l}\right) .
$$

Introduce variables:

and write down (13) in the following form:

$$
\omega_{s c 1}=\omega_{0}+\Omega, \omega_{s c 2}=\omega_{0}-\Omega,
$$

$$
\begin{aligned}
& \tilde{s}_{p}\left(t_{k-1, l}, \mathrm{x}_{k-1}\right)=0,5 A \tilde{h}_{c, p}\left(t_{k-1, l}-\tau_{k-1}\right) \times \\
& \times\left(\sin \left(\omega_{s c 1} t_{k-1, l}+\tilde{\varphi}_{k-1}, l-\Omega \tau_{d, k-1}\right)-\sin \left(\omega_{s c 2} t_{k-1, l}+\tilde{\varphi}_{k-1, l}+\Omega \tau_{d, k-1}\right)\right) .
\end{aligned}
$$

With account of (14) write down (12) in the following form

$$
\begin{gathered}
\frac{1}{\sigma_{n}^{2}} \sum_{l=1}^{N_{p}} y_{k-1, l} \tilde{l}_{p}\left(t_{k-1, l}, \mathrm{x}_{k-1}\right)=\frac{A}{2 \sigma_{n}^{2}} \sum_{l=1}^{N} y_{k-1, l} \tilde{h}_{c, p}\left(t_{k-1, l}-\tau_{k-1}\right) \sin \left(\omega_{s c 1} t_{k-1, l}+\tilde{\varphi}_{k-1, l}-\Omega \tau_{d, k-1}\right)- \\
-\frac{A}{2 \sigma_{n}^{2}} \sum_{l=1}^{N} y_{k-1, l} \tilde{h}_{c, p}\left(t_{k-1, l}-\tau_{k-1}\right) \sin \left(\omega_{s c 2} t_{k-1, l}+\tilde{\varphi}_{k-1, l}+\Omega \tau_{d, k-1}\right) .
\end{gathered}
$$

The presentation already contains the correlation processing at subcarrier frequencies $\omega_{s c 1}$ and $\omega_{s c 2}$ that we will use for further specialization of processing algorithms in the tracking system.

Using trigonometric transformations, we will represent (15) in the following form:

$$
\begin{gathered}
\frac{1}{\sigma_{n}^{2}} \sum_{l=1}^{N} y_{k-1, l} \tilde{s}_{p}\left(t_{k-1, l}, \mathrm{x}_{k-1}\right)=\left(Q_{s c l, k}\left(\tau_{k-1}\right)-Q_{s c 2, k}\left(\tau_{k-1}\right)\right) \cos \left(\Omega \tau_{d, k-1}\right)- \\
-\left(I_{s c l, k}\left(\tau_{k-1}\right)+I_{s c 2, k}\left(\tau_{k-1}\right)\right) \sin \left(\Omega \tau_{d, k-1}\right),
\end{gathered}
$$

where

$$
\begin{aligned}
& I_{s c l, k}\left(\tau_{k-1}\right)=\frac{A}{2 \sigma_{n}^{2}} \sum_{l=1}^{N} y_{k-1, l} \tilde{h}_{c, p}\left(t_{k-1, l}-\tau_{k-1}\right) \cos \left(\omega_{s c 1} t_{k-1, l}+\tilde{\varphi}_{k-1, l}\right), \\
& Q_{s c 1, k}\left(\tau_{k-1}\right)=\frac{A}{2 \sigma_{n}^{2}} \sum_{l=1}^{N} y_{k-1, l} \tilde{h}_{c, p}\left(t_{k-1, l}-\tau_{k-1}\right) \sin \left(\omega_{s c 1} t_{k-1, l}+\tilde{\varphi}_{k-1, l}\right), \\
& I_{s c 2, k}\left(\tau_{k-1}\right)=\frac{A}{2 \sigma_{n}^{2}} \sum_{l=1}^{N} y_{k-1, l} \tilde{h}_{c, p}\left(t_{k-1, l}-\tau_{k-1}\right) \cos \left(\omega_{s c 2} t_{k-1, l}+\tilde{\varphi}_{k-1, l}\right), \\
& Q_{s c 2, k}\left(\tau_{k-1}\right)=\frac{A}{2 \sigma_{n}^{2}} \sum_{l=1}^{N} y_{k-1, l} \tilde{h}_{c, p}\left(t_{k-1, l}-\tau_{k-1}\right) \sin \left(\omega_{s c 2} t_{k-1, l}+\tilde{\varphi}_{k-1, l}\right) .
\end{aligned}
$$

The formulas (16) describe correlators that process the input signal at subcarrier frequencies $\omega_{s c 1}$ and $\omega_{s c 2}$. In the filtration algorithm (8) we shall use the correlators (14), in which the reference signals are taken with extrapolated 
valuations of delays $\tilde{\tau}_{k-1}$ and $\tilde{\tau}_{d, k-1}$, i.e. - when inserting (16) to (8) it is necessary to conduct the change $\tau_{k-1} \rightarrow \tilde{\tau}_{k-1}, \quad \tau_{d, k-1} \rightarrow \tilde{\tau}_{d, k-1}$

Introduce

$$
X_{k}^{2}\left(\tau_{k-1}\right)=\left(Q_{s c 1, k}\left(\tau_{k-1}\right)-Q_{s c 2, k}\left(\tau_{k-1}\right)\right)^{2}+\left(I_{s c 1, k}\left(\tau_{k-1}\right)+I_{s c 2, k}\left(\tau_{k-1}\right)\right)^{2},
$$

and represent (15) in the following form:

$$
\begin{gathered}
\frac{1}{\sigma_{n}^{2}} \sum_{l=1}^{N} y_{k-1, l} \tilde{s}_{p}\left(t_{k-1, l}, \mathrm{x}_{k-1}\right)=X_{k}\left(\tau_{k-1}\right) \times \\
\times\left(\frac{Q_{s c 1, k}\left(\tau_{k-1}\right)-Q_{s c 2, k}\left(\tau_{k-1}\right)}{X_{k}\left(\tau_{k-1}\right)} \cos \left(\Omega \tau_{d, k-1}\right)-\frac{I_{s c 1, k}\left(\tau_{k-1}\right)+I_{s c 2, k}\left(\tau_{k-1}\right)}{X_{k}\left(\tau_{k-1}\right)} \sin \left(\Omega \tau_{d, k-1}\right)\right) .
\end{gathered}
$$

Specify

$$
\begin{gathered}
\cos \left(\psi\left(\tau_{k-1}\right)\right)=\frac{Q_{s c 1, k}\left(\tau_{k-1}\right)-Q_{s c 2, k}\left(\tau_{k-1}\right)}{X_{k}\left(\tau_{k-1}\right)}, \\
\sin \left(\psi\left(\tau_{k-1}\right)\right)=\frac{-\left(I_{s c 1, k}\left(\tau_{k-1}\right)+I_{s c 2, k}\left(\tau_{k-1}\right)\right)}{X_{k}\left(\tau_{k-1}\right)}, \\
\operatorname{tg}\left(\psi\left(\tau_{k-1}\right)\right)=\frac{-\left(I_{s c 1, k}\left(\tau_{k-1}\right)+I_{s c 2, k}\left(\tau_{k-1}\right)\right)}{Q_{s c 1, k}\left(\tau_{k-1}\right)-Q_{s c 2, k}\left(\tau_{k-1}\right)}
\end{gathered}
$$

and represent (19) in the following form:

$$
\frac{1}{\sigma_{n}^{2}} \sum_{l=1}^{N} y_{k-1, l} \tilde{s}_{p}\left(t_{k-1, l}, \mathbf{x}_{k-1}\right)=X_{k}\left(\tau_{k-1}\right) \cos \left(\Omega \tau_{d, k-1}-\psi\left(\tau_{k-1}\right)\right) .
$$

The quasioptimal filtration equations (8) sometimes are called "extended Kalman filters". The form of such a presentation is not adequately corresponded to the presentation of classic tracking system, in which there are discriminator and smoothing filter. The discriminator is understood to mean the device, the output process of which contains information about mismatch between true value of signal parameter that is tracked and its estimating value that is formed in tracking system. In general, the discriminator on $\lambda$ parameter of signal is described with the following formula:

$$
u_{d \lambda, k}=\left.\frac{\partial \tilde{F}_{k}\left(\mathrm{x}_{k-1}\right)}{\partial \lambda}\right|_{\mathrm{x}_{k-1}=\tilde{\mathrm{x}}_{k-1}}
$$

In the considering task the parameters being tracked are the $\tau$ signal delay and additional variable $\tau_{d}$.

Taking into account the fact that functions $\tilde{F}_{k}\left(\mathrm{x}_{k-1}\right)$ are defined with formula (11), for which the presentation (21) was obtained, we will write down the formula for discriminator on $\tau$ delay (delay discriminator):

$$
u_{d \tau, k}=\left.\frac{\partial}{\partial \tau}\left[\frac{1}{\sigma_{n}^{2}} \sum_{l=1}^{N} y_{k-1, l} \tilde{s}_{p}\left(t_{k-1, l}, \mathbf{x}_{k-1}\right)\right]\right|_{\tilde{x}_{k-1}} \approx \frac{\partial X_{k}\left(\tilde{\tau}_{k-1}\right)}{\partial \tau} \cos \left(\psi\left(\tilde{\tau}_{k-1}\right)-\Omega \tilde{\tau}_{d, k-1}\right)
$$

When writing down (22) we have admitted the assumption about independence of $\psi$ from $\tau_{k-1}$.

We can consider the simplified variant of $\tau$ delay discriminator, of insert $\cos \left(\Omega \tau_{k-1}-\psi\left(\tau_{k-1}\right)\right) \approx 1$ to $(22)$. In 
such a case, the $\tau$ delay discriminator can be described by simpler formula:

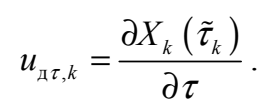

When dealing with navigation equipment the calculation of derivative on $\tau$ in (22) is interchanged with calculation of finite differences:

$$
\frac{\partial X_{k}\left(\tilde{\tau}_{k}\right)}{\partial \tau} \approx \frac{X_{k}\left(\tilde{\tau}_{k}+\Delta \tau / 2\right)-X_{k}\left(\tilde{\tau}_{k}-\Delta \tau / 2\right)}{\Delta \tau},
$$

where $\Delta \tau-$ the mismatch between reference signals of correlators (16), i.e. - between the ranging codes $\tilde{h}_{c, p}\left(t_{k-1, l}-\tau_{k-1}\right)$ of the present reference signals.

The structural scheme of $\tau$ delay discriminator is presented in Figure 2, where $I_{s c 1 E, k}\left(\tilde{\tau}_{k-1}\right), I_{s c 2 E, k}\left(\tilde{\tau}_{k-1}\right)$, $Q_{s c 1 E, k}\left(\tilde{\tau}_{k-1}\right), Q_{s c 2 E, k}\left(\tilde{\tau}_{k-1}\right), I_{s c 1 L, k}\left(\tilde{\tau}_{k-1}\right), I_{s c 2 L, k}\left(\tilde{\tau}_{k-1}\right), Q_{s c L L, k}\left(\tilde{\tau}_{k-1}\right), Q_{s c 2 L, k}\left(\tilde{\tau}_{k-1}\right)-$ are the advance and delayed components of correlators (16).

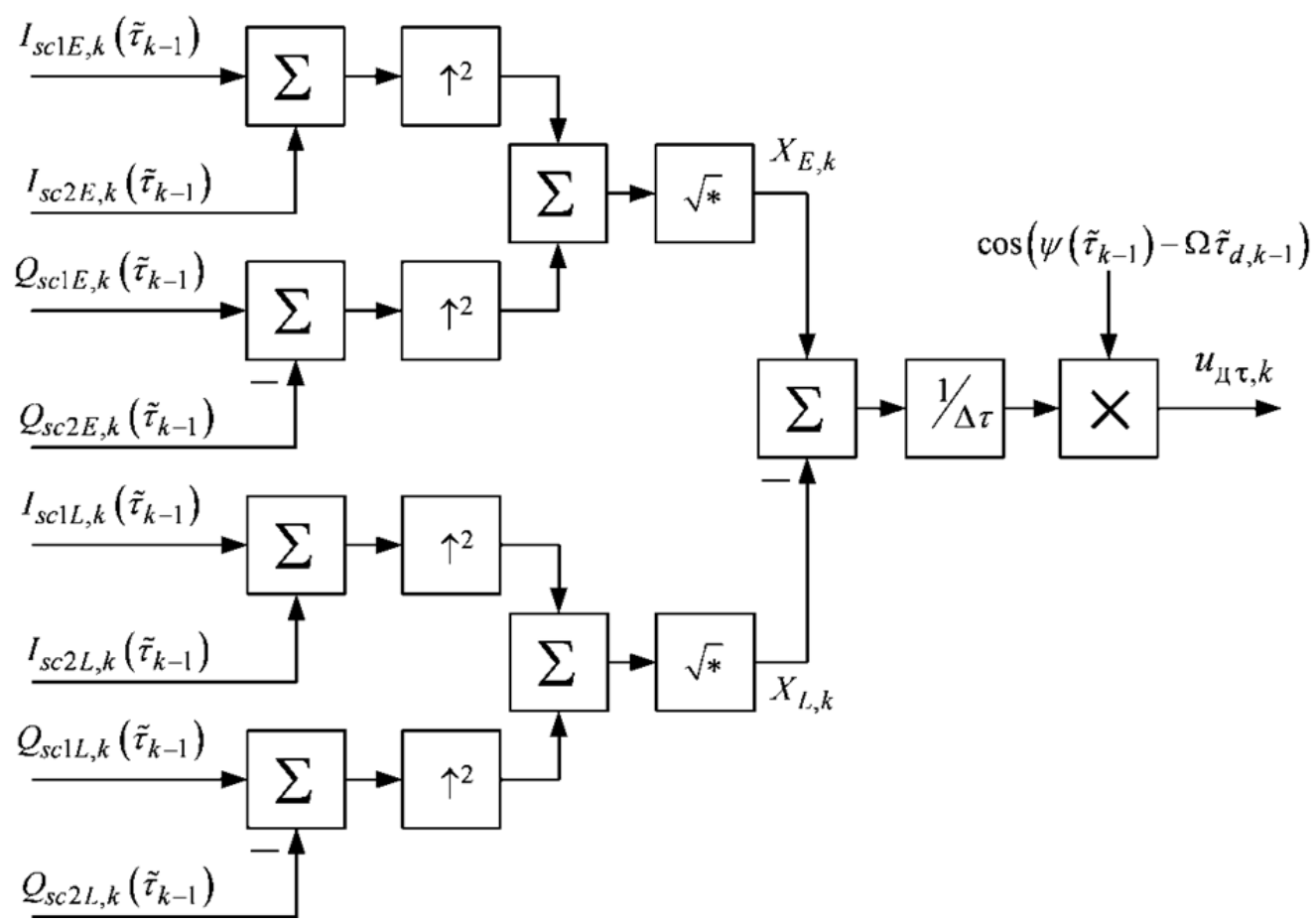

Figure 2. The structural scheme of $\tau$ delay discriminator

Now let us consider the discriminator on additional variable $\tau_{d}$

$$
u_{d \tau_{d}, k}=\left.\frac{\partial}{\partial \tau_{d}}\left[\frac{1}{\sigma_{n}^{2}} \sum_{l=1}^{N_{p}} y_{k-1, l} \tilde{s}_{p}\left(t_{k-1, l}, \mathrm{x}_{k-1}\right)\right]\right|_{\tilde{x}_{k-1}}=\Omega X_{k}\left(\tilde{\tau}_{k-1}\right) \sin \left(\psi\left(\tilde{\tau}_{k-1}\right)-\Omega \tilde{\tau}_{d, k-1}\right)
$$

The structural scheme of $\tau_{d}$ delay discriminator is shown in Figure 3. 


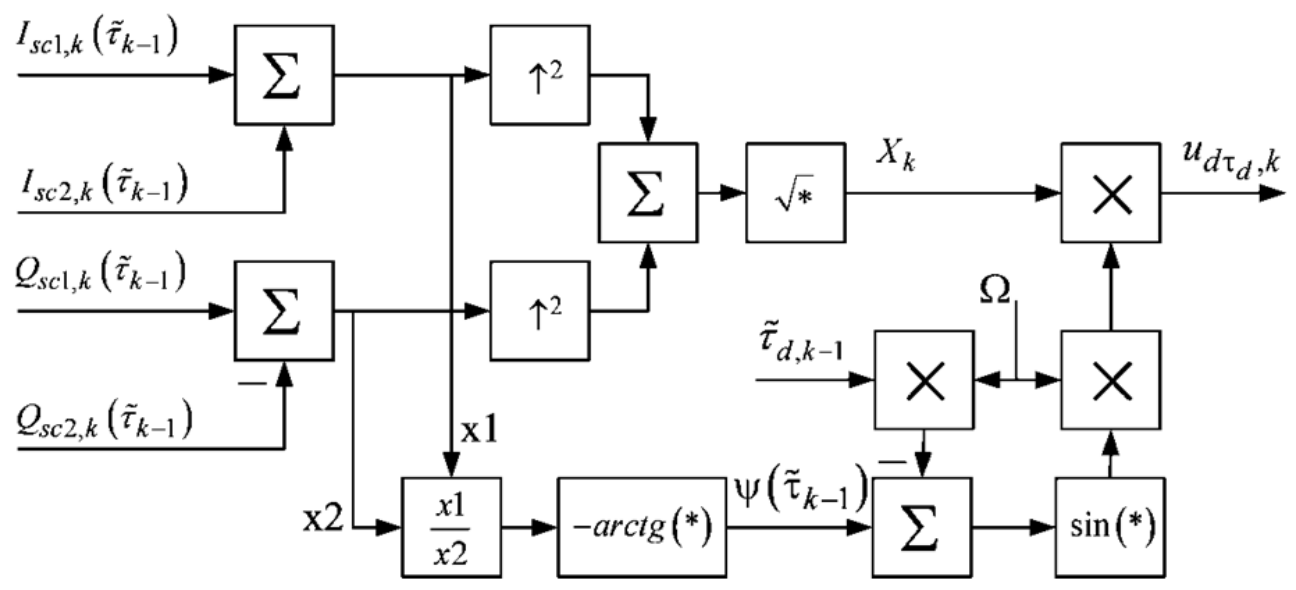

Figure 3. The structural scheme of discriminator on $\tau_{d}$ additional variable

Function (24) - is being periodical on $\tau_{d}$ and its stable zeros correspond to the condition $\psi\left(\tilde{\tau}_{k-1}\right)-\Omega \tilde{\tau}_{d, k-1}=2 \pi m, \quad m=0, \pm 1, \ldots$. or

$$
\hat{\tau}_{d}=\frac{1}{\Omega}\left(\psi\left(\tilde{\tau}_{k}\right)-2 \pi m\right)=\hat{\tau}_{d 0}+T_{\Omega} m,
$$

where $T_{\Omega}=2 \pi / \Omega \quad$ - the fluctuation period of subcarrier frequency; $\tilde{\tau}_{d 0}=\psi\left(\tilde{\tau}_{k}\right) / \Omega$.

The formula (25) depends on the parameter $m$ defining the ambiguousness of estimation. To eliminate this ambiguousness in the additional variable method (Tikhonov, V. I., \& Harisov, V. N., 2004) we have taken the algorithm of estimation of the present parameter in the following form:

$$
\hat{m}=\arg \left(\min _{m}\left(\left|\hat{\tau}-\hat{\tau}_{d}\right|\right)\right)=\arg \left(\min _{m}\left(\left|\hat{\tau}-\left(\hat{\tau}_{d 0}+T_{\Omega} m\right)\right|\right)\right) .
$$

For the resulting valuation of delay $\hat{\tau}_{\text {res }}$ there is formula:

$$
\hat{\tau}_{\text {res }}=\hat{\tau}_{d 0}(\hat{\tau})+\hat{m} T_{\Omega} .
$$

In the equation of optimal filtration (8) there is derivative $\frac{\partial \tilde{F}_{k}\left(\tilde{x}_{k-1}\right)}{\partial \mathrm{x}}$, for which we can write down the following expression:

$$
\frac{\partial \tilde{F}_{k}\left(\tilde{\mathrm{x}}_{k-1}\right)}{\partial \mathrm{x}}=\left|\frac{\partial \tilde{F}_{k}\left(\tilde{\mathrm{x}}_{k-1}\right)}{\partial \tau} \quad \frac{\partial \tilde{F}_{k}\left(\tilde{\mathrm{x}}_{k-1}\right)}{\partial \tau_{d}}\right|^{T}=\left|u_{d \tau, k} \quad u_{d \tau_{d}, k}\right|^{T} .
$$

With account of (27) the equation (8) describing the tracking system takes the following form:

$$
\hat{\mathrm{x}}_{k-1}=\tilde{\mathrm{x}}_{k-1}+\mathrm{D}_{\mathrm{x}, k-1}\left|u_{d \tau, k} \quad u_{d \tau_{d}, k}\right|^{T} .
$$

The structural scheme of signal delay tracking system is presented in Figure 4, where $z^{-1}$ means the block of delay per working cycle of tracking system, the block « $\tau$ correction» performs the formation of resulting delay estimation $\hat{\tau}_{\text {res }}$ in accordance with algorithm (26). 


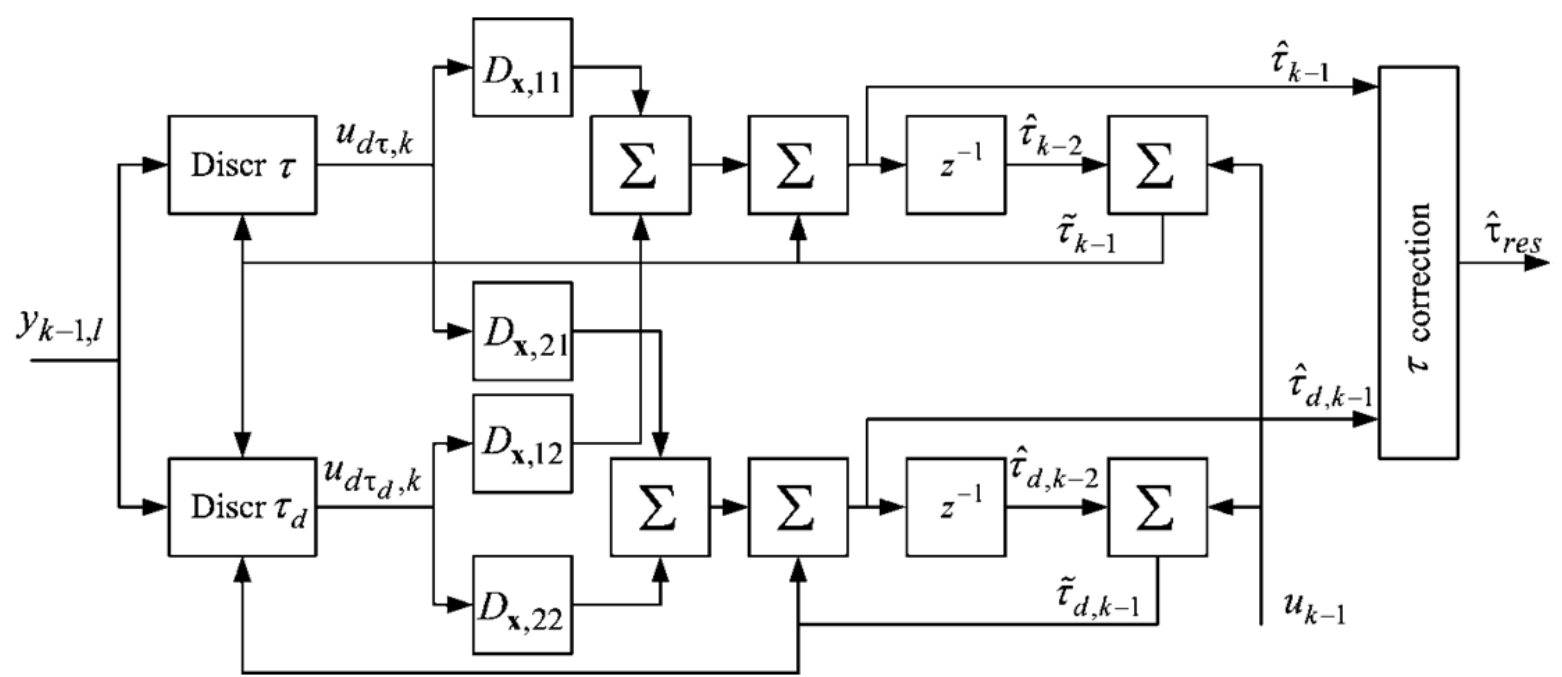

Figure 4. The structural scheme of signal delay tracking system

Contrary to the known signals delay tracking systems in the scheme of tracking presented in Figure 4 in one tracking loop there are two delay discriminators $\tau$ and $\tau_{d}$, thus in this loop there will be two estimations $\hat{\tau}$ and $\hat{\tau}_{d}$, from which the resulting estimation of $\hat{\tau}_{\text {res }}$ signal delay is formed out of the loop of the tracking system.

\section{Results and Discussion}

\subsection{Analysis of Discriminator Curves}

One of the peculiarities of synthesized delay filtering algorithm is a presence of two time discriminators, one of which (22) extracts information from delay of ranging code $h_{c, p}\left(t_{k-1, l}\right)$, while the second (23) extracts information from delay of digital sinusoid $h_{d s}\left(t_{k-1, l}\right)$. Considering it, we will analyse the statistical characteristics of the present discriminators.

We shall calculate mathematical expectations of correlation sums (16) with account of substituting.

Perform substituting $\varphi_{k-1} \rightarrow \tilde{\varphi}_{k-1}, \quad \omega_{D, k-1} \rightarrow \tilde{\omega}_{D, k-1}$

$$
\begin{gathered}
E\left[I_{s c l, k}\right]=\bar{I}_{s c 1, k}=\frac{A^{2} T}{2 \pi N_{0}} \rho_{p}\left(\varepsilon_{\tau}\right) \sin \left(\varepsilon_{\varphi, k}+\varepsilon_{\omega, k} T / 2-\Omega \tau_{d, k-1}\right) \operatorname{sinc}\left(\varepsilon_{\omega, k} T / 2\right)= \\
=\frac{q_{c / n_{0}} T}{\pi} \rho_{p}\left(\varepsilon_{\tau}\right) \sin \left(\varepsilon_{\varphi, k}+\varepsilon_{\omega, k} T / 2-\Omega \tau_{d, k-1}\right) \operatorname{sinc}\left(\varepsilon_{\omega, k} T / 2\right), \\
E\left[Q_{s c 1, k}\right]=\bar{Q}_{s c l, k}=\frac{A^{2} T}{2 \pi N_{0}} \rho_{p}\left(\varepsilon_{\tau}\right) \cos \left(\varepsilon_{\varphi, k}+\varepsilon_{\omega, k} T / 2-\Omega \tau_{d, k-1}\right) \operatorname{sinc}\left(\varepsilon_{\omega, k} T / 2\right)= \\
=\frac{q_{c / n_{0}} T}{\pi} \rho_{p}\left(\varepsilon_{\tau}\right) \cos \left(\varepsilon_{\varphi, k}+\varepsilon_{\omega, k} T / 2-\Omega \tau_{d, k-1}\right) \operatorname{sinc}\left(\varepsilon_{\omega, k} T / 2\right), \\
E\left[I_{s c 2, k}\right]=\bar{I}_{s c 2, k}=\frac{-A^{2} T}{2 \pi N_{0}} \rho_{p}\left(\varepsilon_{\tau}\right) \sin \left(\varepsilon_{\varphi, k}+\varepsilon_{\omega, k} T / 2+\Omega \tau_{d, k-1}\right) \operatorname{sinc}\left(\varepsilon_{\omega, k} T / 2\right)= \\
=\frac{-q_{c / n_{0}} T}{\pi} \rho_{p}\left(\varepsilon_{\tau}\right) \sin \left(\varepsilon_{\varphi, k}+\varepsilon_{\omega, k} T / 2+\Omega \tau_{d, k-1}\right) \operatorname{sinc}\left(\varepsilon_{\omega, k} T / 2\right),
\end{gathered}
$$




$$
\begin{aligned}
E\left[Q_{s c 2, k}\right]= & \bar{Q}_{s c 2, k}=\frac{-A^{2} T}{2 \pi N_{0}} \cos \left(\varepsilon_{\varphi, k}+\varepsilon_{\omega, k} T / 2+\Omega \tau_{d, k-1}\right) \operatorname{sinc}\left(\varepsilon_{\omega, k} T / 2\right)= \\
= & \frac{-q_{c / n_{0}} T}{\pi} \cos \left(\varepsilon_{\varphi, k}+\varepsilon_{\omega, k} T / 2+\Omega \tau_{d, k-1}\right) \operatorname{sinc}\left(\varepsilon_{\omega, k} T / 2\right), \\
& \text { where } \rho_{p}\left(\varepsilon_{\tau}\right)=\frac{1}{T / 2} \int_{0}^{T} \tilde{h}_{c, p}\left(t-\tau_{k-1}\right) \tilde{h}_{c, p}\left(t-\tilde{\tau}_{k-1}\right) d t
\end{aligned}
$$

— correlation function of ranging code $h_{c, p}\left(t_{k-1, l}\right)$ of pilot signal component;

$$
\operatorname{sinc}(x)=\frac{\sin (x)}{x}
$$

$$
\begin{aligned}
& \varepsilon_{\varphi, k}=\varphi_{k-1}-\tilde{\varphi}_{k-1}-\text { tracking error; } \\
& \varepsilon_{\omega, k}=\omega_{k-1}-\tilde{\omega}_{k-1}-\text { Doppler frequency shift tracking error; } \\
& \varepsilon_{\zeta, k}=\tau_{k-1}-\tilde{\tau}_{k-1}-\text { ranging code delay tracking error } h_{c, p}\left(t_{k-1, l}\right) ; \\
& q_{c / n_{0}}=\frac{A^{2}}{2 N_{0}}=\frac{P_{c}}{N_{0}}-\text { ratio of signal power to noise one-way power spectral density. }
\end{aligned}
$$

Calculate the average value of envelope's square (17)

$$
\bar{X}_{k}^{2}=\left(\frac{2}{\pi} q_{c / n_{0}} T\right) \rho_{p}^{2}\left(\varepsilon_{\tau, k}\right) \cos ^{2}\left(\varepsilon_{\varphi, k}+\varepsilon_{\omega, k} T / 2\right) \operatorname{sinc}^{2}\left(\varepsilon_{\omega, k} T / 2\right) .
$$

Subsequently, we can write down:

$$
\bar{X}_{k}\left(\varepsilon_{\tau}\right)=\frac{2}{\pi} q_{c / n_{0}} T\left|\rho_{p}\left(\varepsilon_{\tau, k}\right) \cos \left(\varepsilon_{\varphi, k}+\varepsilon_{\omega, k} T / 2\right) \operatorname{sinc}\left(\varepsilon_{\omega, k} T / 2\right)\right| .
$$

The discriminator curve of ranging code (22) delay discriminator when exchanging of delay differential with finite increment in case of small level of errors $\varepsilon_{\varphi} \approx 0, \quad \varepsilon_{\omega} \approx 0$ is the following:

$$
U_{\text {discr, }, \tau}\left(\varepsilon_{\tau}\right)=\frac{2 q_{c / n_{0}} T}{\pi \Delta \tau}\left(\left|\rho_{p}\left(\varepsilon_{\tau}-\Delta \tau / 2\right)\right|-\left|\rho_{p}\left(\varepsilon_{\tau}+\Delta \tau / 2\right)\right|\right) .
$$

The formula for discriminator curve (29) aligns with similar formula for discriminator curve taken for delay discriminator designed for processing signal with modulation BPSK(1)

The aperture of discriminator curve (in case of conventionally used value $\left.\Delta \tau=\tau_{c}\right) \Delta_{\tau}= \pm 3 / 2 \tau_{c} \quad( \pm 440 \mathrm{~m})$. The slope of discriminator curve is equal to

$$
S_{\text {discr, },}=\frac{4 q_{c / n_{0}} T}{\pi \tau_{c}^{2}} .
$$

When concluding we have used some approximations, i.e. - when digital sinusoid was changed to natural sinusoid, what is being the definite approximation. The normalized discriminator value depending on standardized delay error $\varepsilon_{\tau_{n}}=\varepsilon_{\tau} / \tau_{c}$ without use of specified approximations calculated by means of computer you can see of Figure 5. 


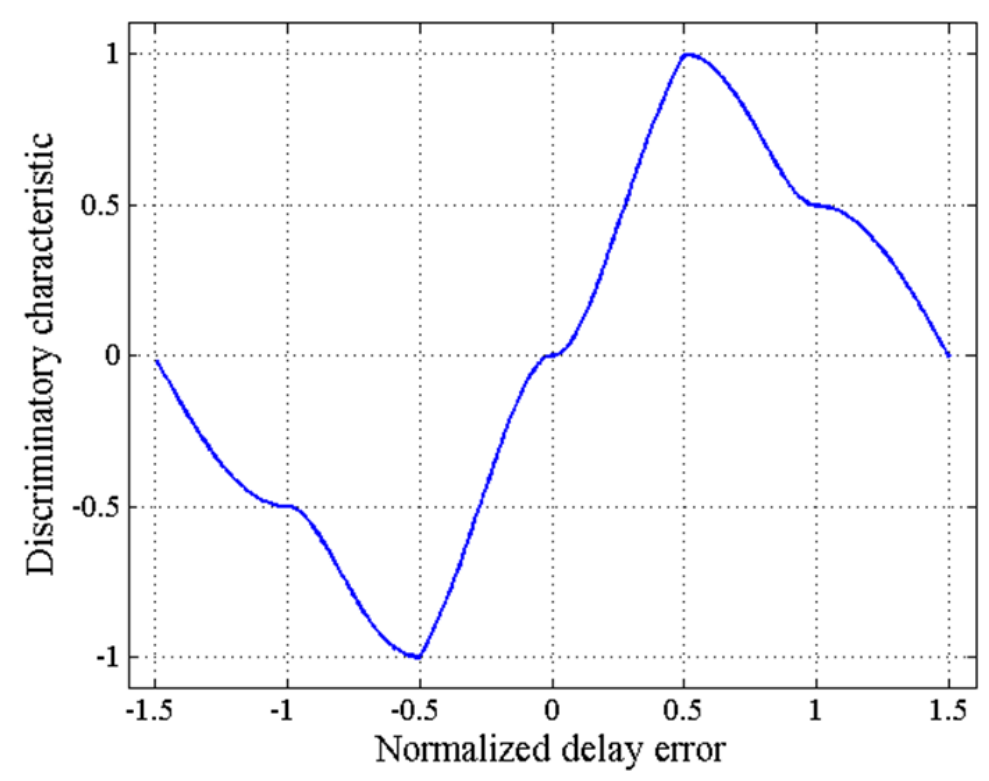

Figure 5. Standardized ranging code delay error

For dispersion of fluctuating component at the output of discriminator, we can use the following formula (using $\left.\Delta \tau=\tau_{c}\right)$

$$
D_{u_{d}, f l}=\frac{q_{c / n_{0}} T}{2 \tau_{c}^{2}},
$$

which is similar to the formula for dispersion of fluctuating component at the output of discriminator working in response to signal with modulation BPSK (1) (Perov, A. I., 2012).

Another important characteristic of quality of tracking systems is being the dispersion of noise of delay discriminator lead to the measuring parameter (Perov, A. I., 2012). We set it as $D_{e f, \tau}$. The present characteristics is univalently linked to the Rao-Cramer's low limit for dispersion when measuring the parameter of signal, i.e. it defines potential accuracy of measurement of correspond parameter (Tikhonov, V. I., \& Harisov, V. N., 2004). For the examining ranging code delay discriminator write

$$
D_{e f, \tau}=\frac{D_{u_{d}, f l}}{S_{d, \tau}^{2}}=\frac{\pi^{2} \tau_{c}^{2}}{32 q_{c / n_{0}} T} .
$$

From the comparison of the present formula with similar formula for dispersion of delay discriminator noise in processing at carrier frequency it follows that for the systems with processing at subcarrier frequency the dispersion is 1.23 times bigger than for system with processing at carrier frequency.

Further, we will consider discriminator on additional variable (24). Its discriminator value can be described with formula

$U_{\text {д, } \tau}\left(\varepsilon_{\tau}\right) \approx \Omega \bar{X}_{k} \sin \left(\Omega \varepsilon_{\tau_{d}}\right)=\frac{2 \Omega q_{c / n_{0}} T}{\pi}\left|\rho_{p}\left(\varepsilon_{\tau, k}\right) \cos \left(\varepsilon_{\varphi, k}+\varepsilon_{\omega, k} T / 2\right) \operatorname{sinc}\left(\varepsilon_{\omega, k} T / 2\right)\right| \sin \left(\Omega \varepsilon_{\tau_{d}}\right)$ In case of small errors $\varepsilon_{\varphi} \approx 0, \quad \varepsilon_{\omega} \approx 0$ we can write down the approximate expression:

$$
U_{\text {म, } \tau_{d}}\left(\varepsilon_{\tau_{d}}\right) \approx \frac{2 \Omega q_{c / n_{0}} T}{\pi}\left|1-\frac{\left|\varepsilon_{\tau}\right|}{\tau_{c}}\right| \sin \left(\Omega \varepsilon_{\tau}\right)=\frac{2 \Omega q_{c / n_{0}} T}{\pi}\left|1-\frac{\left|\varepsilon_{\tau}\right|}{\tau_{c}}\right| \sin \left(2 \pi \frac{\varepsilon_{\tau_{d}}}{\tau_{c}}\right) .
$$

The discriminator (32) on additional variable $\tau_{d}$ is alternating with period $T_{\tau_{d}}=\tau_{c}$. In Figure 6 there is normalized discriminator curve as the function standardized error function $\varepsilon_{\tau_{d}} / \tau_{c}$. 


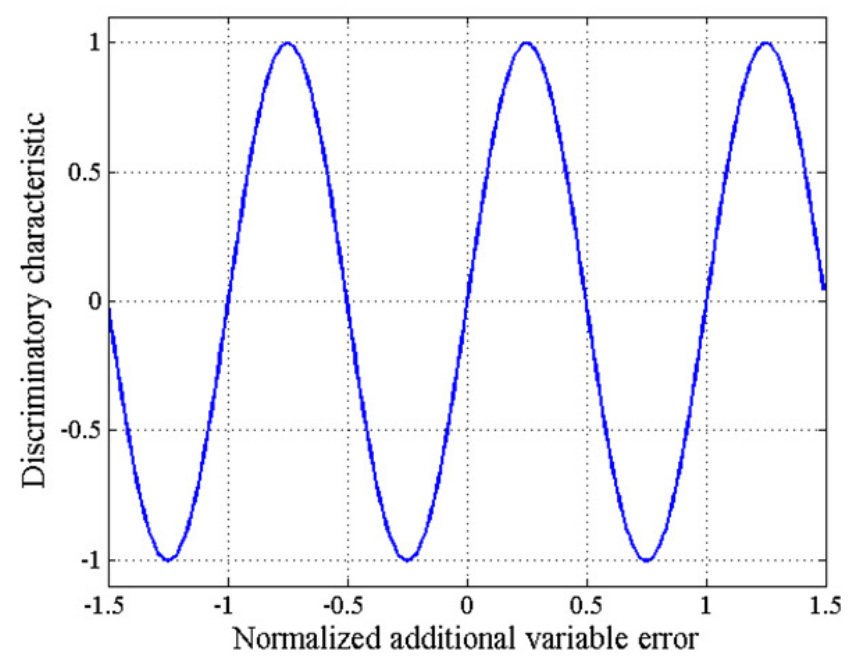

Figure 6. The standardized curve on additional variable $\tau_{d}$

The aperture of discriminator curve makes $\Delta_{\tau}= \pm 0,5 \tau_{c}( \pm 146.6 \mathrm{~m})$. The slope of discriminator curve is equal to

$$
S_{\text {discr, }, \tau_{d}}=\frac{4 q_{c / n_{0}} T \Omega}{\tau_{c}} .
$$

From the comparison of (33) with (30), it follows that if $\Delta \tau=\tau_{c}$; the slope of discriminator curve on additional variable is $2 \pi^{2}$ times bigger than the slope of discriminator curve on ranging code delay.

The statistical characteristics of discriminators of ranging code delay and additional variable can be used for simplification of dispersion equations (9). The essence of such simplification lies in substitution instead of derivative $\frac{\partial}{\partial \mathrm{x}}\left(\frac{\partial \tilde{F}_{k}\left(\tilde{\mathrm{x}}_{k}\right)}{\partial \mathrm{x}}\right)^{T}$ its average value $E\left[\frac{\partial}{\partial \mathrm{x}}\left(\frac{\partial \tilde{F}_{k}\left(\tilde{\mathrm{x}}_{k}\right)}{\partial \mathrm{x}}\right)^{T}\right]$. Performing such averaging we will get:

$$
\begin{gathered}
\left.E\left[\frac{\partial}{\partial \mathrm{x}}\left(\frac{\partial \tilde{F}_{k}\left(\tilde{\mathrm{x}}_{k}\right)}{\partial \mathrm{x}}\right)^{T}\right]\right|_{\tilde{\mathrm{x}}_{k}=\mathrm{x}_{k}}=E\left[\frac{\partial}{\partial \mathrm{x}}\left|u_{d \tau, k} \quad u_{d \tau_{d}, k}\right|\right]||_{\tau}=0, \varepsilon_{\tau_{d}}=0= \\
\quad=\frac{\partial}{\partial \mathrm{x}}\left(\begin{array}{c}
U_{d i s c r, \tau}\left(\varepsilon_{\tau}\right) \\
U_{\pi, \tau_{d}}\left(\varepsilon_{\tau_{d}}\right)
\end{array}\right) \mid \varepsilon_{\tau}=0, \varepsilon_{\tau_{d}}=0 \\
=-\left|\begin{array}{cc}
S_{\text {discr, },} & 0 \\
0 & S_{\text {disc }, \tau_{d}}
\end{array}\right|=-\mathrm{S}_{\text {discr, }, \tau} .
\end{gathered}
$$

Inserting the present formula to the dispersion equations belonging to (9), we will right down the simplified formulas:

$$
\tilde{\mathrm{D}}_{\mathrm{x}, k-1}=\mathrm{D}_{\mathrm{x}, k-2}+\mathrm{G} D_{\xi} \mathrm{G}^{T}, \quad \mathrm{D}_{\mathrm{x}, k-1}^{-1}=\tilde{\mathrm{D}}_{\mathrm{x}, k-1}^{-1}+\mathrm{S}_{d i s c r, \tau} .
$$

The present dispersion equations will be used later, when simulation modelling the synthesized tracking system.

From the analysis of dispersion equations (34), it follows that in steady-state regime $\mathrm{D}_{\mathrm{x}}(1,1)=\mathrm{D}_{\mathrm{x}}(1,2)=\mathrm{D}_{\mathrm{x}}(2,1)=\mathrm{D}_{\mathrm{x}}(2,2)=D_{\tau, \text { steady }}$. In so doing the filtration equation will be

$$
\hat{\mathrm{x}}_{k-1}=\tilde{\mathrm{x}}_{k-1}+\mathrm{P} D_{\tau, s t e a d y} u_{d \Sigma \tau, k}, \quad \tilde{\mathrm{x}}_{k-1}=\mathrm{F} \hat{\mathrm{x}}_{k-2}+\mathrm{P} u_{k-1},
$$

where 


$$
u_{d \Sigma \tau, k}=u_{d \tau, k}+u_{d \tau_{d}, k}
$$

- is the summarized discriminator.

In steady-state regime, $\hat{\tau} \rightarrow \hat{\tau}_{d}$ we can consider the discriminator curve of summarized discriminator $u_{d \Sigma \tau, k}$ as the function of one generalized error $\varepsilon_{\tau}$. The standardized discriminator curve of summarized discriminator as the function of standardized error $\varepsilon_{\tau_{n}}=\varepsilon_{\tau} / \tau_{c}$ is presented in Figure 8.

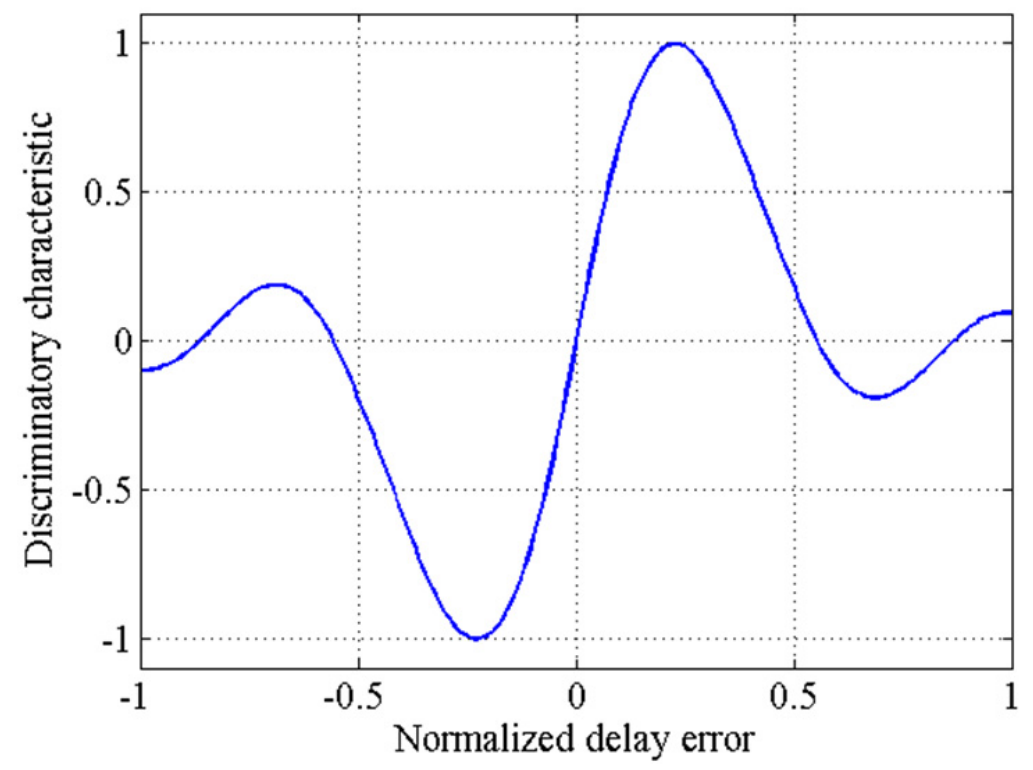

Figure 7. Standardized discriminator curve of summarized

The aperture of discriminator curve makes $\Delta_{\tau}= \pm 0,555 \tau_{c}( \pm 163 \mathrm{~m})$. The slope of discriminator curve is equal to

$$
S_{\text {discr }, \Sigma \tau}=\frac{4 q_{c / n_{0}} T}{\pi \tau_{c}^{2}}\left(1+2 \pi^{2}\right),
$$

i.e. much bigger than the slope of discriminator curve only on ranging code delay.

\subsection{Synthesized Tracking System Modelling}

To test the working capacity and characteristics of synthesized tracking system for delay of L1OC GLONASS signal pilot component with processing at subcarrier frequencies we have conducted its modelling by means of digital computer in the environment of MATLAB simulation. The modelling was carried out with use of statistical equivalents method upon the following conditions: $\tilde{q}_{c / n_{0}}=\log \left(q_{c / n_{0}}\right)=45 \mathrm{dbHz} ; T=8 \mathrm{~ms}$.

Firstly we will examine the case when delay $\tau_{k}$ is described with equation (5), the valuation of Doppler frequency shift $\tilde{\omega}_{D, k}$ is known to us, the tracking system is described with transient equation (28), (34). Let $D_{\xi}=4 T \mathrm{~m}^{2}$. In Figures 8,9 there are realizations of $\tau$ delay tracking error and additional variable $\tau_{d}$ tracking error taken as the result of modelling. The dashed red lines in these Figures mean the limits of confidential intervals $\pm 3 \sigma_{\tau}$ and $\pm 3 \sigma_{\tau_{d}}$, where $\sigma_{\tau}$ и $\sigma_{\tau_{d}}$ - the least square value of $\tau$ delay tracking error and additional variable $\tau_{d}$ tracking error calculated by equations of Riccati (34). 


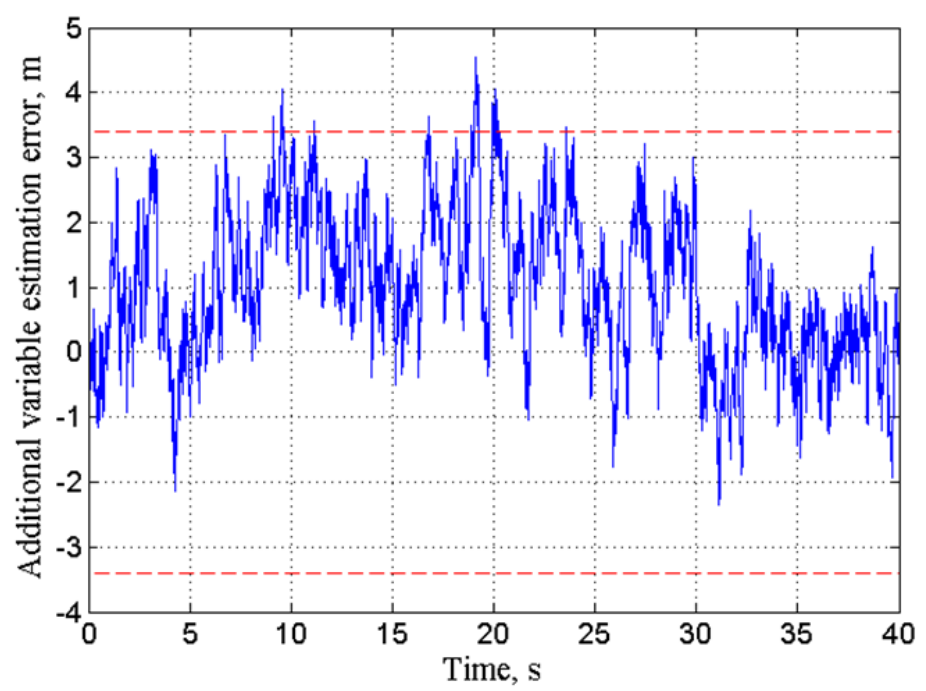

Figure 8. $\tau$-delay tracking error

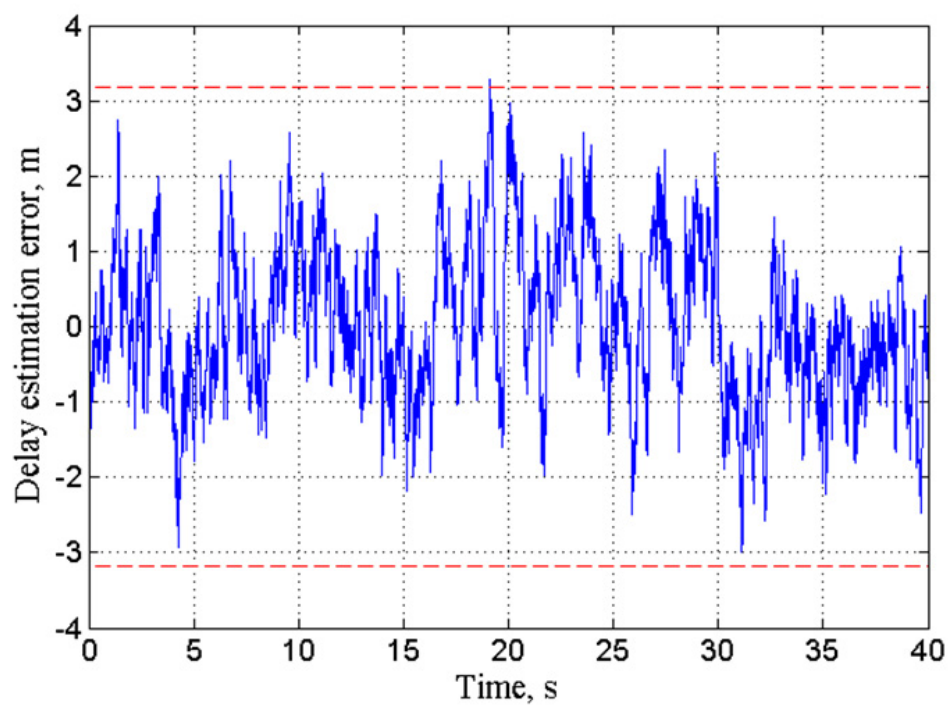

Figure 9. $\tau_{d}$-additional variable tracking error

In the set regime the band-pass range of tracking system is $\Delta f_{D L L}=0.86 \mathrm{~Hz}$. The least square value of delay tracking error calculated by realization of Figure 8 is $\sigma_{\tau, \text { calc }}=1.53 \mathrm{~m}$, while the least square value of additional variable tracking error calculated by realization of Figure $9 \sigma_{\tau_{d}, \text { calc }}=1.0 \mathrm{M}$. In such a way, there is quite long transient process when measuring $\tau$ delay.

In Figures 10, 11 there are similar tracking errors in case of weaker dynamics of delay change. 


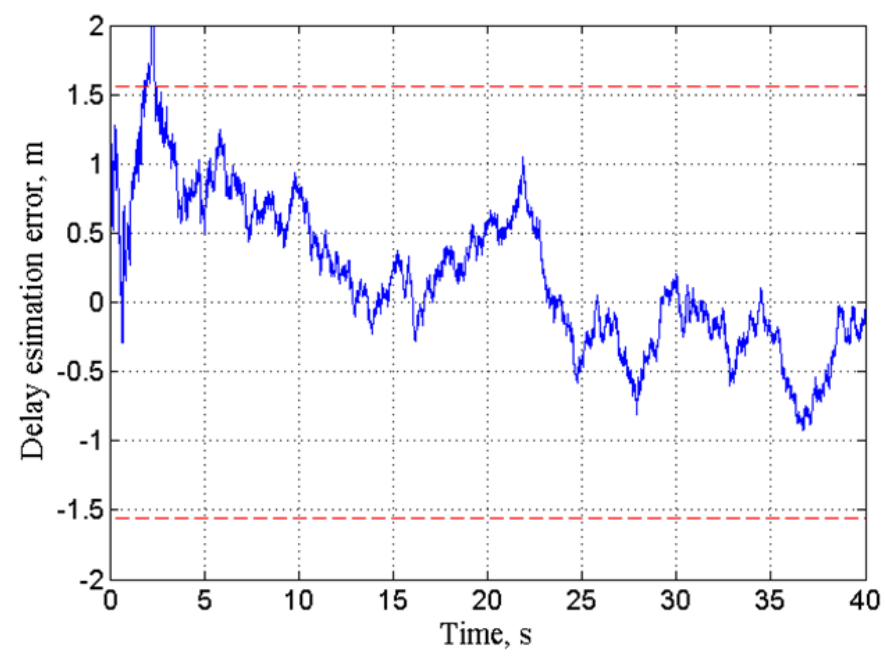

Figure 10. $\tau$-delay tracking error

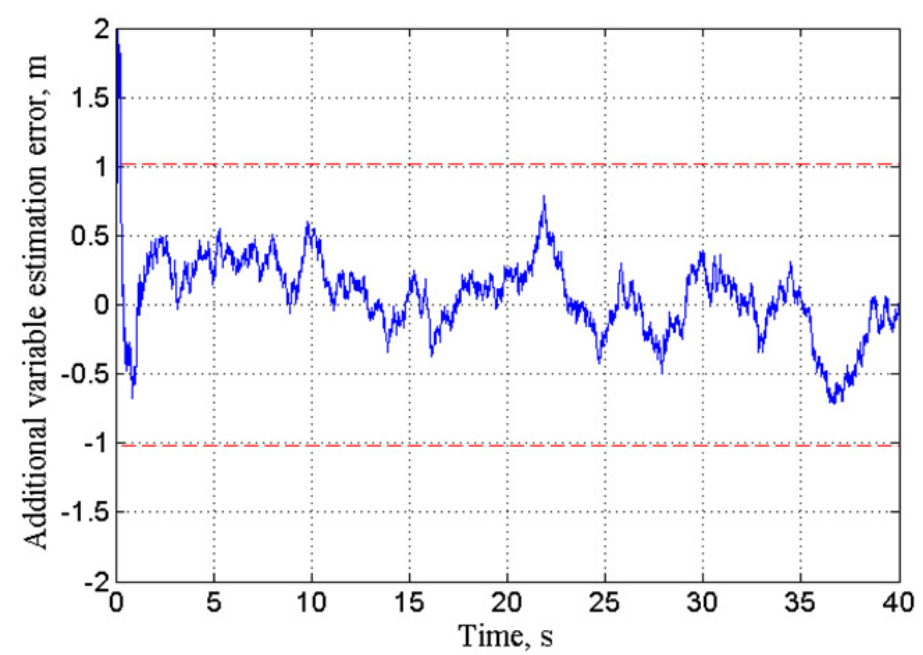

Figure 11. $\tau_{d}$ additional variable tracking error

In the steady-state regime the bandwidth of range tracking system is $\Delta f_{D L L}=0.088 \mathrm{~Hz}$. The mean square value (rms) of delay tracking error calculated by realization of Figure 10 is $\sigma_{\tau, \text { calc }}=1.53 \mathrm{~m}$, while the mean square value of additional variable tracking error calculated by realization of Figure 11 is $\sigma_{\tau_{d}, \text { calc }}=0.32 \mathrm{~m}$.

We shall note that in the tracking system built on the principle of signals processing at carriers frequency (Perov, A. I., \& Harisov, V. N., 2010) the least square value of GLONASS signal pilot component delay tracking error makes $\sigma_{\tau, \text { calc }}=1.72 \mathrm{~m}$ for dynamic change of delay, while in case of weak dynamic of delay it makes $\sigma_{\tau, \text { calc }}=$ $0.59 \mathrm{~m}$. Thus, the synthesized system with processing at subcarrier frequencies provides $1.5-1.7$ times reduction of the mean square tracking error in set regime.

\section{Conclusion}

In this paper, we have synthesized the quasioptimal algorithm of L1OC GLONASS signal pilot component delay filtering with processing at subcarrier frequencies with use of the theory of optimal filtering of information processes and the method of additional variable. 
We have taken the equations of optimal filtering and their representation in the form of tracking system including delay discriminator, additional variable discriminator and smoothing filter.

It is shown that synthesized discriminators with processing of pilot component of L1OC signal at subcarrier frequencies have structures that differ from the structures of known delay discriminators with processing at carrier frequency.

There are also structural schemes of synthesized discriminators. We have calculated the discriminator and fluctuation characteristics of delay and additional variable discriminators. In addition, it is shown that discriminator curves have stable equilibrium points. In the steady-state regime in the tracking system we can point out the summarized discriminator, the slope of discriminator curve of which is $2 \pi^{2}$ bigger than in delay discriminator only for ranging code, what provides increase of tracking accuracy.

We have also listed the results of simulation modelling of synthesized tracking system. It is specified that in optimal tracking system at signal/noise ratio equal to $40 \mathrm{dbHz}$ the least square value of signal delay tracking error makes $\sim 0,35 \mathrm{~m}$ (rms) for low-dynamic consumer and $\sim 1,0$ degrees - for high-dynamic consumer. In the tracking system with processing at subcarrier frequencies in the steady-state regime there is $1,5-1,7$ times reduction of the mean square tracking error in comparison with similar tracking error in the system with processing at carrier frequency.

\section{References}

BeiDou Navigation Satellite Navigation System. Signal in Space Interface Control Document (2011).

Chen, S., Theil, K. H., \& Kleusberg, A. (2006). Implementation and Analysis of Acquisition and Tracking Algorithms for BOC Signals/ ION GNSS 19th International Technical Meeting of the Satellite Division, 26-29, September.

David de Castro, Diez, J., \& Fernandez, A. (2006). Unambiguous Low-Complexity BOC Tracking Technique. ION GNSS 19th International Technical Meeting of the Satellite Division, 26-29.

European GNSS (GALILEO). (2010). Open Service. Signal in Space Interface Control Document.

Fante, R. L. (2003). Unambiguous Tracker for GPS Binary-Offset-Carrier Signals, Proceedings of the 59th Annual Meeting of The Institute of Navigation and CIGTF 22nd Guidance Test Symposium, 23-25 June, Albuquerque, NM, The Institute of Navigation, 141-145.

Fine, P., \& Wilson, W. (1999). Tracking Algorithm for GPS Offset Carrier Signals, Proceedings of the 1999 National Technical Meeting, San Diego, CA, The Institute of Navigation, 671-676.

GLONASS. (4th ed.) (2010). In Perov A. I., \& Harisov V. N. (Eds.), Principles of generation and operation (p800). Moscow: Radiotehnika

Gyu-In, J., Sung-Hyuck, I., \& Byung-Hyun, L. (2007). Optimal Code and Carrier Tracking Loop Design for Galileo BOC (1,1). ION GNSS 20th International Technical Meeting of the Satellite Division, 25-28, 759-768.

Heiries, V., Roviras, D., Ries, L., \& Calmettes, V. (2004). Analysis of Non Ambiguous BOC Signal Acquisition Performance, ION.

Hodgart, M. S., \& Blunt, P. D. (2007). The Optimal Dual Estimate Solution for Robust Tracking of Binary Offset Carrier (BOC) Modulation. ION GNSS 20th International Technical Meeting of the Satellite Division, 25-28, September 2007.

Hodgart, M. S., Weiler, R. M., \& Unwin, M. (2008). A Triple Estimating Receiver of Multiplexed Binary Offset Carrier (MBOC) Modulated Signals. ION GNSS 21st. International Technical Meeting of the Satellite Division, 16-19, September 2008, 877-886.

Interface Specification GPS Space Segment/ User Segment. IS-GPS-200F, 21 September 2011.

Interface specification GPS Space Segment/User Segment L1C Interfaces (2011). IS-GPS-800B, 21.

Jovancevic, A., Ganguly, S., Sirpatil, B., Kirchner, M., \& Zigic, S. (2007). Real-Time Software Based Block Processing M-code Receiver. ION GNSS 20th International Technical Meeting of the Satellite Division, 25-28.

Jovanovic, A., Mongredien, C., Tawk, Y., Botteron, C., \& Farine, P. A. (2012.) Two-Step Galileo El CBOC Tracking Algorithm: When Reliability and Robustness Are Keys. International Journal of Navigation and Observation, Volume 2012. 
Martin, N., Leblond, V., Guilloyel, G., \& Heiries, V. (2003). Analysis of Non Ambiguous BOC Signal Acquisition Performance», ION.

Musso, M., Cattoni, A. F., \& Regazzoni, C. S. (2006). A New Fine Tracking Algorithm for Binary Offset Carrier Modulated Signals. ON GNSS 19th International Technical meeting of the Satellite Division, 26-29 September.

Perov, A. I. (2012). Basics of satellite radio navigation systems construction. Moscow: Radiotehnika, $240 \mathrm{p}$.

Perov, A. I. (2012). Methods and algorithms of optimal signal detection in the equipment of satellite radio navigation systems' users. Moscow: Radiotehnika, $240 \mathrm{p}$.

Tikhionov, V. I., \& Kharisov, V. N. (1984). Integral synchronization in radiotechnical systems. Radio engineering, 39(4), 3-10.

Tikhionov, V. I., \& Kharisov, V. N. (2004). Statistic analysis and synthesis wireless device and systems. Moscow: Radio y svyaz, 608p.

Understanding GPS principles and applications (2006). Ed. by E. D. Kaplan, C. J. Hegarty, published by Artech House Inc., Norwood, Massachusetts.

Vinogradov, A. A., Perkov, A. E., \& Rodchenko, A. O. (2009). Estimation algorithms of GNSS advanced signal delay with BOC-modulation based on supplementary variable technique. Radio engineering, pp. 99-104.

\section{Copyrights}

Copyright for this article is retained by the author(s), with first publication rights granted to the journal.

This is an open-access article distributed under the terms and conditions of the Creative Commons Attribution license (http://creativecommons.org/licenses/by/3.0/). 